\title{
Pré-natal odontológico: acessibilidade e ações ofertadas pela atenção básica de Vitória da Conquista-BA
}

\author{
Prenatal dental care: accessibility and shares offered \\ by primary care in Vitória da Conquista-BA
}

\author{
Suélem Maria Santana Pinheiro Ferreira' \\ Érica Silva Pinheiro" \\ Rogério Vieira Silva" \\ Jinária Fernandes Silva" \\ Larissa Dias Batista" \\ Carolle Gomes Fernandes"
}

\begin{abstract}
RESUMO
Objetivo: Buscou-se caracterizar a acessibilidade e ações executadas no pré-natal odontológico de Vitória da Conquista (BA). Métodos: Estudo transversal, com 268 mulheres, gestantes (89,7\%) e puérperas (10,3\%), usuárias da Atenção Básica municipal. Procedeu-se a coleta de dados com formulário, englobando atividades prestadas pela equipe de saúde bucal às gestantes. Os dados foram tabulados e analisados através do SPSS, obtendo-se frequências absolutas e relativas. Resultados: As mulheres estavam inseridas no pré-natal, realizando frequentemente uma a três consultas $(37,2 \%)$, apesar disso, foi expressiva a ausência de contato com dentista (74,6\%). Apenas 25,4\% foram inseridas em ações odontológicas, predominantemente no serviço público $(61,0 \%)$, dentre as quais $39,0 \%$ com visitas pontuais por dor. A participação em ações educativas com dentistas foi baixa (12; 17,9\%), nas quais 41,7\% relataram não ter oportunidade de emitir opinião. Informações importantes para a saúde bucal do bebê foram pouco trabalhadas, tais como: vantagens do aleitamento materno $(4,5 \%)$, uso de chupeta $(36,4 \%)$, quando e como realizar a higiene bucal do bebê (47,0\% e 40,9\% respectivamente), riscos de xaropes açucarados (13,6\%) e uso de açúcar/mel (28,8\%). Conclusão: Não se observou consolidação do pré-natal odontológico, devido à baixa participação em ações assistenciais, preventivas ou de promoção da saúde bucal. Ademais, intervenções frequentemente se direcionaram para alívio da dor, fora da dimensão integral.
\end{abstract}

Palavras-Chave: Gestantes. Saúde Bucal. Cuidado Pré-natal.

\begin{abstract}
Objectives: We seek to characterize the accessibility and the actions performed in the prenatal dental care in the city of Vitória da Conquista (BA). Methods: Therefore, we conducted a cross-sectional study with 268 women, pregnant women $(89.7 \%)$ and postpartum women $(10.3 \%)$, users of the municipal primary care. Data collection was performed with forms, encompassing activities provided by the dental health team for pregnant women. The data were tabulated and analyzed using SPSS, thus obtaining absolute and relative frequencies. Results: Women were included in the prenatal, often performed one to three consultations $(37.2 \%)$, although it was significant the absence of contact with the dentist $(74.6 \%)$. Only $25.4 \%$ were inserted in dental actions, predominantly in the public service $(61.0 \%)$, of which $39.0 \%$ with consultations caused by pain. The participation in educational activities with dentists was low $(12 ; 17.9 \%)$, in which $41.7 \%$ reported not had opportunity of express an opinion. Important information for the oral health of the baby were little worked, such as advantages of breastfeeding (4.5\%), pacifier use (36.4\%), when and how to perform oral hygiene baby $(47.0 \%$ and $40.9 \%$ respectively), risks of sweet substances $(13.6 \%)$ and use of sugar / honey $(28.8 \%)$. Conclusions: The prenatal dental care did not appear as consolidated practice. There was not observed the consolidation of prenatal dental, due to low participation in health care activities, preventive or of oral health promotion. Moreover, often interventions are directed toward relief of pain, without of the integral dimension.
\end{abstract}

Key words: Pregnant Women. Oral Health. Prenatal Care.

Universidade do Estado da Bahia (UNEB), Salvador/BA - Brasil

"Faculdade Independente do Nordeste (FAINOR), Vitória da Conquista/BA - Brasil 


\section{INTRODUÇÃO}

O período gestacional é permeado por mudanças fisiológicas, que impõem aos profissionais de saúde o desafio de conhecêlas, visando ofertar um cuidado diferenciado e adequado, num momento tão singular. Como parte desse processo, a saúde bucal da gestante, pode sofrer interferências de alterações hormonais, enjoos frequentes, aumento da frequência alimentar e dificuldade em realizar higiene oral, favorecendo o aparecimento de alterações bucais. Ademais, o estado da saúde bucal apresentado durante a gestação pode influenciar na saúde geral da gestante, bem como na saúde geral e bucal do bebê1.

Apesar da maior susceptibilidade para desenvolvimento de algumas doenças bucais, há relato na literatura de que gestantes vão menos ao dentista ${ }^{2}$, sobretudo pela influência de crenças e mitos, ainda arraigados no imaginário popular, que associam intervenções odontológicas na gestação, com prejuízos para o bebê ${ }^{3}$.

Desta forma, ressalta-se a necessidade da participação do dentista em programas de prénatal, a partir do pré-natal odontológico. Nesse contexto, o desafio inicial é apoiar a gestante no tocante à sua insegurança em procurar o serviço odontológico, a partir do contato com respeito às crenças, e tentar romper barreiras, por meio do estabelecimento e/ou consolidação do vínculo. Por último, o pré-natal odontológico, deve ser pensando dentro de uma linha de cuidado pautada na integralidade, acolhimento e corresponsabilização, possibilitando a execução de ações assistenciais, prevenção de doenças bucais, promoção de saúde bucal à gestante, e orientação de cuidados bucais que devem ser tomados com o bebê $\hat{e}^{4}$.

Elias et al. ${ }^{5}$ afirmam que as gestantes podem apresentar problemas periodontais relacionados ao próprio período gestacional, como a gengivite gravídica. Além disso, na literatura há relato de que a doença periodontal pode ser fator de risco para o parto pré-termo e/ou baixo peso ao nascer ${ }^{6}$.

Conforme tem sido descrito na literatura, a cárie dentária é a doença bucal mais prevalente na gestante e pode causar diversos outros problemas, como a perda do elemento dentário e a consequente necessidade de prótese. ${ }^{7}$ Soma-se a isso, a evidência de que cuidados preventivos durante a gravidez influenciam na ocorrência ou não de lesões cariosas em bebês ${ }^{8}$.

Alguns estudos apontam que a mulher, durante a gestação, tem se mostrado mais disposta a adquirir novos conhecimentos e propícia a modificar hábitos, que possam influenciar a saúde e o cuidado com o bebê, tornando-se assim um grupo estratégico para educação em saúde sob abordagem multidisciplinar ${ }^{3,7}$. Nesse caso, oportunamente os profissionais envolvidos no cuidado do prénatal devem estabelecer compartilhamento de agendas, que facilitem o desenvolvimento de grupos operativos como esse público, e a equipe de saúde bucal deve buscar nesses espaços a ocasião favorável para fortalecer o pré-natal odontológico, em todas as suas dimensões ${ }^{9}$.

No Brasil, os esforços para melhorar a qualidade da atenção à gestante, se destacam desde meados da década de 1990, com vistas à implementação de políticas de saúde na perspectiva da integralidade ${ }^{10}$. Como exemplo importante, a Política Nacional de Saúde Bucal define que ao iniciar o atendimento pré-natal, a gestante deverá passar por consulta odontológica que minimamente avalie condições de tecidos moles, doença periodontal, cárie dentária, orientação sobre higiene bucal e alimentação, respeitando o segundo trimestre como melhor fase para intervenções clínicas ${ }^{11}$. Somando-se a isso, o Manual de Pré-Natal de Baixo Risco 2012 traz a assistência odontológica como parte 
integrante da atenção pré-natal. Apesar disso, a atenção odontológica vem sendo pouco abordada e em muitas realidades não está incluída nos cuidados de rotina à gestante ${ }^{12}$.

Atualmente, um novo esforço de ampliação dos cuidados à gestante é demonstrado, a partir da estratégia "Rede Cegonha", que visa estruturar a atenção à saúde maternoinfantil no Brasil e insere o dentista como profissional necessário ao atendimento básico na gestação. Além disso, elenca atribuições desse profissional que envolvem: sensibilização ao pré-natal, amamentação e vacinação; orientações da periodicidade de consultas, estratégias educativas quanto às novas vivências da gestação, cuidados típicos de cada fase e saúde bucal do bebê; avaliação da saúde geral e bucal; identificação de fatores de risco e adequação de meio bucal; tratamento específico, com os cuidados de cada fase gestacional, que eliminem riscos à gravidez e restabeleçam conforto à gestante; atendimento de urgências/emergências; busca ativa de gestantes faltosas; visita domiciliar na gestação e puerpério; suporte à amamentação e cuidados bucais do bebê ${ }^{12}$.

Neste contexto, o presente artigo buscou identificar a inserção da Odontologia no âmbito da atenção pré-natal e caracterizar as ações executadas na Atenção Básica de Vitória da Conquista (BA), entre 2013 e 2014. Como lócus privilegiado para o cuidado pré-natal, e ao contar com a inserção do dentista, a Atenção Básica representa também uma referência para o pré-natal odontológico.

\section{Métodos}

Trata-se de um estudo transversal descritivo, com 268 gestantes e puérperas, residentes no município de Vitória da Conquista, Bahia, Brasil. A rede de atenção, nesse município, é estruturada a partir de 29 Unidades de Saúde da Família (USF), nas quais atuam 39
Equipes de Saúde da Família (ESF), além de 08 Unidades Básicas de Saúde (UBS). Na zona urbana, há 14 USF's, com 23 ESF's, bem como todas as UBS's estão localizadas nessa zona. Por outro lado, o município conta com 37 Equipes de Saúde Bucal (ESB), das quais 14 encontram-se inseridas em USF's urbanas, em sua maioria na proporção 1:2 (uma ESB para 2 ESF), e outras 07 nas UBS's.

O estudo iniciou-se após a aprovação pelo Comitê de Ética em Pesquisa (CEP) da Faculdade Independente do Nordeste, sob parecer 418.773/2013. A participação das entrevistadas esteve condicionada a assinatura de um termo de consentimento livre e esclarecido, e durante toda a construção/ realização da pesquisa foram respeitados os aspectos éticos e legais.

A população de referência foi constituída de gestantes e puérperas, residentes na zona urbana de Vitória da Conquista, que realizavam acompanhamento pré-natal em USF's e UBS's, com equipe de saúde bucal, entre dezembro de 2013 e maio de 2014. Dentre as UBS's, uma não possuía ESB e outras duas estavam em reforma, e por isso foram excluídas do estudo, por outro lado, todas as demais foram incluídas na pesquisa, totalizando 5 UBS's. Entretanto, no caso das USF's, a seleção das participantes foi constituída por amostragem de conglomerados de um único estágio. Nesse sentido, as USF's, que contavam com ESB (total de 14), foram incluídas em um sorteio aleatório, o qual definiu as 5 USF's $(35,7 \%$ do total de USF's) que seriam incluídas na pesquisa. Além disso, através de sorteio, também se definiu a ordem de entrada das USF's e UBS's no estudo (amostragem no tempo). Após essa fase, a cada mês, 1 USF e 1 UBS recebiam os pesquisadores para um período de coleta de 30 dias, no qual todas as gestantes e puérperas que se apresentavam para o acompanhamento prénatal e puerpério, e atendessem aos critérios 
de inclusão (puérperas sem complicações durante a gestação ou após o parto que impediram a amamentação, comparecer para acompanhamento de pré-natal ou puerpério na Unidade Básica durante o período de coleta de dados, aceitação em participar da pesquisa), eram convidadas a participar do estudo.

Os dados foram coletados através de um formulário de pesquisa, aplicado pela técnica direta, com perguntas direcionadas às mulheres pelo entrevistador. No total, quatro entrevistadores, devidamente treinados, participaram da coleta de dados. O formulário abrangeu variáveis referentes à caracterização sociodemográfica da amostra, caracterização da gestação e prénatal, definição e delineamento de ações de promoção, prevenção e reabilitação em saúde bucal, realizadas durante a gestação. As variáveis selecionadas buscaram incorporar informações sobre as diversas facetas que englobam o pré-natal odontológico.

A definição do formato final do formulário de pesquisa foi pautada na realização de um estudo piloto, anterior à coleta de dados, visando a elucidar deficiências, bem como erros de pergunta, e corrigi-los. Este estudo foi realizado em uma USF, da zona urbana, com ESB, definida mediante sorteio. A coleta de dados do estudo piloto aconteceu com toda a população de gestante e puérperas, que buscaram a USF para acompanhamento pré-natal e puerpério, no decorrer de uma semana.

Após a coleta, os dados foram organizados em bancos e analisados através do software $R$ versão 3.2.2. A análise partiu dos princípios da estatística descritiva, e sumarizou os achados a partir de frequências absolutas e relativas. As variáveis contínuas foram transformadas em variáveis nominais, com categorias definidas a partir de pontos de corte da literatura ou da própria distribuição dos dados. Após isso, também foi realizada análise bivariada descritiva e o teste de qui-quadrado de Pearson, com nível de significância de 5\%. Este foi executado para avaliar a associação de variáveis socioeconômicas, demográficas, características gestacionais e uso de serviços odontológicos com o contato com o cirurgiãodentista no período gestacional.

\section{Resultados}

Dentre as 268 gestantes e puérperas incluídas no estudo, a maior parte encontravase sob acompanhamento em UBS's, e no $2^{\circ}$ $(81 ; 30,8 \%)$ ou $3^{\circ}$ trimestre $(111 ; 42,2 \%)$ da gestação. A maioria delas tinha idade entre $20-35$ anos $(69,4 \%)$ e cor parda $(62,2 \%)$. Uma parte considerável das mulheres possuía renda familiar situada entre um e dois salários mínimos (64,4\%). A união estável com o parceiro $(43,4 \%)$ e o casamento $(34,8 \%)$ foram características conjugais da maioria dessas mulheres, que frequentemente relataram não ter filhos $(47,9 \%)$, ou possuir no máximo dois (43,0\%) (Tabela 1).

Quanto à utilização do serviço de saúde bucal, ofertado pelas unidades de saúde da Atenção Básica, verificou-se que a maior parte das mulheres estudadas não era usuária do mesmo $(67,4 \%)$. O acesso a tais serviços foi relatado como difícil para $41,7 \%$ das mulheres, enquanto $55,2 \%$ relataram não os ter sequer procurado. Cabe destacar que essas informações remetem ao período anterior à gestação.

Quando foi avaliada a relação das variáveis sociodemográficas, gestacionais e de uso de serviços com a existência de contato com o cirurgião-dentista na gestação, verificou-se associação apenas para o tipo de unidade de saúde $(p=0,001)$, idade gestacional $(p=0,014)$, uso do serviço odontológico da unidade $(p<0,001)$ e dificuldade de acesso ao mesmo $(p=0,005)$, sendo as duas últimas variáveis referidas ao período anterior à gestação. 
Tabela 1: Caracterização sociodemográfica, gestacional e uso do serviço odontológico de gestantes e puérperas atendidas na Atenção Básica. Vitória da Conquista-BA, Brasil. 2013-2014.

\begin{tabular}{|c|c|c|c|c|c|c|c|}
\hline \multirow[b]{3}{*}{ VARIÁVEIS } & \multicolumn{4}{|c|}{$\begin{array}{c}\text { Contato com o dentista durante a } \\
\text { gestação }\end{array}$} & & & \multirow[b]{3}{*}{$p^{2}$} \\
\hline & \multicolumn{2}{|c|}{ Sim } & \multicolumn{2}{|c|}{ Não } & \multicolumn{2}{|c|}{ Total } & \\
\hline & $\mathbf{n}$ & $\%$ & $\mathrm{n}$ & $\%$ & $\mathrm{n}^{1}$ & $\%$ & \\
\hline \multicolumn{8}{|l|}{$\begin{array}{l}\text { Tipo de estabelecimento } \\
(\mathrm{n}=268)\end{array}$} \\
\hline Unidade básica de saúde & 28 & 17,8 & 129 & 82,2 & 157 & 58,6 & 0,001 \\
\hline Unidade de saúde da família & 40 & 36,0 & 71 & 64,0 & 111 & 41,4 & \\
\hline \multicolumn{8}{|l|}{ Faixa Etária $(\mathrm{n}=268)$} \\
\hline$<20$ & 11 & 18,6 & 48 & 81,4 & 59 & 22,0 & 0,378 \\
\hline $20-35$ & 50 & 26,9 & 136 & 73,1 & 186 & 69,4 & \\
\hline$>35$ & 7 & 30,4 & 16 & 69,6 & 23 & 8,6 & \\
\hline \multicolumn{8}{|l|}{ Raça/cor (n=267) } \\
\hline Preta & 13 & 24,1 & 41 & 75,9 & 54 & 20,2 & 0,492 \\
\hline Parda & 39 & 23,5 & 127 & 76,5 & 166 & 62,2 & \\
\hline Branca & 15 & 31,9 & 32 & 68,1 & 47 & 17,6 & \\
\hline \multicolumn{8}{|l|}{ Idade gestacional $(n=263)$} \\
\hline $1^{\circ}$ trimestre & 5 & 11,4 & 39 & 88,6 & 44 & 16,7 & 0,014 \\
\hline $2^{\circ}$ trimestre & 18 & 22,2 & 63 & 77,8 & 81 & 30,8 & \\
\hline $3^{\circ}$ trimestre & 39 & 35,1 & 72 & 64,9 & 111 & 42,2 & \\
\hline Puerpério & 6 & 22,2 & 21 & 77,8 & 27 & 10,3 & \\
\hline \multicolumn{8}{|l|}{ Renda Familiar ( $n=267$ ) } \\
\hline$<1$ salário & 11 & 15,7 & 59 & 84,3 & 70 & 26,2 & 0,077 \\
\hline 1-2 salários & 51 & 29,7 & 121 & 70,3 & 172 & 64,4 & \\
\hline 3-5 salários & 6 & 24,0 & 19 & 76,0 & 25 & 9,4 & \\
\hline \multicolumn{8}{|l|}{ Situação conjugal ( $n=267$ ) } \\
\hline Solteira & 9 & 17,0 & 44 & 83,0 & 53 & 43,4 & 0,145 \\
\hline Casada & 32 & 34,4 & 61 & 65,6 & 93 & 34,8 & \\
\hline Divorciada & 1 & 25,0 & 3 & 75,0 & 4 & 19,9 & \\
\hline Viúva & 0 & 0 & 1 & 100 & 1 & 1,5 & \\
\hline União consensual/estável & 26 & 22,4 & 90 & 77,6 & 116 & 0,4 & \\
\hline \multicolumn{8}{|l|}{ Número de Filhos $(n=265)$} \\
\hline Nenhum & 23 & 21,3 & 85 & 78,7 & 108 & 40,4 & 0,443 \\
\hline Até 2 filhos & 37 & 30,1 & 86 & 69,9 & 123 & 46,1 & \\
\hline 3-4 filhos & 7 & 23,3 & 23 & 76,7 & 30 & 11,2 & \\
\hline$>=5$ filhos & 1 & 16,7 & 5 & 83,3 & 6 & 2,2 & \\
\hline \multicolumn{8}{|l|}{$\begin{array}{l}\text { Uso do serviço odontológico } \\
\text { da unidade }(n=264)\end{array}$} \\
\hline Sim & 41 & 47,7 & 45 & 52,3 & 86 & 32,6 & $<0,001$ \\
\hline Não & 27 & 15,2 & 151 & 84,8 & 178 & 67,4 & \\
\hline \multicolumn{8}{|l|}{$\begin{array}{l}\text { Dificuldade de acesso ao } \\
\text { serviço odontológico da } \\
\text { unidade }(n=120)^{3}\end{array}$} \\
\hline Sim & 12 & 24,0 & 38 & 76,0 & 50 & 41,7 & 0,005 \\
\hline Não & 34 & 48,6 & 36 & 52,4 & 70 & 58,3 & \\
\hline
\end{tabular}

1 Apesar da amostra ser composta de 268 mulheres, para algumas questões houve perdas (indivíduo se recusou a responder a pergunta ou relatou não saber respondê-la) durante o processo de coleta de dados, o que resultou em "n" menores para algumas variáveis."

2 Teste qui-quadrado de Pearson

3 Do total da amostra, 148 mulheres $(55,2 \%)$ nunca buscou o serviço odontológico da unidade 
As mulheres entrevistadas estavam inseridas no acompanhamento pré-natal/ puerperal regularmente, e realizaram frequentemente uma a três consultas pré-natal $(37,2 \%)$, o que, em alguns casos, pode ser reflexo da fase gestacional na qual ocorreu a coleta de dados. Apesar disso, foi expressiva a ausência de contato com o dentista na gestação $(74,6 \%)$, com apenas 68 mulheres $(25,4 \%)$ inseridas em ações odontológicas, em até duas consultas $(39 ; 66,1 \%)$ predominantemente no serviço público $(36 ; 61,0 \%)$, dentre as quais 23 mulheres $(39,0 \%)$ por visitas pontuais em decorrência de dor (Tabela 2).

Quanto às consultas realizadas durante - período gestacional, foi apontada resolubilidade total dos problemas bucais em 27 casos (45,8\%) e parcial em 21 (35,6\%). $\mathrm{Na}$ maior parte das vezes, foram criadas na consulta oportunidades para que a gestante expressasse suas queixas $(47 ; 81,0 \%)$, apesar disso, menos da metade das mulheres teve oportunidade de emitir opinião sobre o tratamento odontológico a ser realizado (26; $45,6 \%$ ).

A Tabela 3 mostra que a participação em ações educativas com dentistas, na gestação, foi relatada por apenas 12 mulheres $(17,9 \%)$, e destas apenas $7(58,3 \%)$ relataram que estas ações foram exclusivamente direcionadas para o grupo de gestantes. Nestas ações educativas, apesar de 7 mulheres $(41,7 \%)$ relatarem não ter oportunidade para emitir opinião, sobre os temas tratados pelo dentista, a maior parte (8; 66,7\%) conseguiu resposta para suas dúvidas. De maneira geral, as mulheres que tiveram contato com o dentista, quer em ações educativas individuais ou coletivas, foram pouco informadas sobre aspectos importantes para a saúde bucal do bebê, pois foi pouco frequente o relato de orientações, tais como: vantagens do aleitamento materno (3; 4,5\%), uso e implicações da chupeta ortodôntica (24; $36,4 \%$ ), quando e como realizar a higiene bucal do bebê $(31(47,0 \%)$ e $27(40,9 \%)$ respectivamente), cuidados no uso de xaropes açucarados $(9 ; 13,6 \%)$ e oferta de açúcar/mel (19; 28,8\%).

\section{Discussão}

Os principais achados desta investigação demonstram uma realidade preocupante, quando se trata de cuidados odontológicos à saúde bucal do binômio mãe-filho, pois aludem que essa linha de cuidado não está consolidada, o que contribui para a não realização do pré-natal odontológico. Cabe destacar que o percentual de contato com o cirurgião-dentista foi cerca de duas vezes maior entre as usuárias de unidade de saúde da família, quando comparadas com a Atenção Básica tradicional, demonstrando que essa estratégia de organização da Atenção Básica, tem contribuído de forma mais enfática para mudança do modelo de atenção à saúde bucal. Além disso, entre as mulheres que já utilizavam o serviço odontológico da unidade de saúde, ou que não relataram dificuldades de acesso a este serviço, previamente à gestação, os percentuais de contato com o cirurgião-dentista no período gestacional foram, respectivamente, cerca de três e duas vezes maiores que para as mulheres que não usavam o serviço odontológico ou relataram barreiras no acesso ao mesmo, antes da gestação.

Quando se analisa o desenvolvimento do pré-natal odontológico, duas linhas de ações devem sincronizar-se: as ações de promoção/prevenção e as ações assistenciais individuais ${ }^{13}$. Em ambos os aspectos, verificouse uma deficiência considerável na amostra estudada. Apesar disso, a maioria relatou não ter dificuldade em realizar o agendamento com o dentista, ou nem sequer ter procurado o serviço odontológico, diferente de outras situações, nas quais o profissional cria barreiras à realização do atendimento de gestantes ${ }^{14}$. 
Tabela 2: Caracterização do pré-natal odontológico na Atenção Básica: participação em ações clínicas. Vitória da Conquista-BA, Brasil. 2013-2014¹.

\begin{tabular}{|c|c|c|}
\hline VARIAVÉIS & $\mathbf{n}$ & $\%$ \\
\hline \multicolumn{3}{|c|}{ Número de consultas pré-natal realizadas $(n=266)^{*}$} \\
\hline Nenhuma & 20 & 7,5 \\
\hline 1-3 consultas & $\begin{array}{r}99 \\
85\end{array}$ & 37,2 \\
\hline$>=6$ consultas & 62 & 23,3 \\
\hline \multicolumn{3}{|c|}{ Algum contato com o dentista na gestação $(n=268)^{*}$} \\
\hline Sim & 68 & 25,4 \\
\hline Não & 200 & 74,6 \\
\hline \multicolumn{3}{|c|}{ Consulta com o dentista na gestação $(n=67)^{\star *}$} \\
\hline Sim & 59 & 88,1 \\
\hline Não & 8 & 11,9 \\
\hline \multicolumn{3}{|c|}{ Local da consulta odontológica $(n=59)^{* * *}$} \\
\hline Unidade de saúde & 36 & 61,0 \\
\hline Consultório particular & 21 & 35,6 \\
\hline Ambos & 2 & 3,4 \\
\hline \multicolumn{3}{|c|}{ Motivo da consulta odontológica $(n=59)^{\star * *}$} \\
\hline Dor & 23 & 39,0 \\
\hline Iniciar tratamento & 9 & 15,3 \\
\hline Continuar tratamento & 10 & 16,9 \\
\hline Ações preventivas & 17 & 28,8 \\
\hline \multicolumn{3}{|c|}{ Número de consultas odontológicas $(n=59)^{\star * *}$} \\
\hline Até 2 consultas & 39 & 66,1 \\
\hline$\geq 3$ consultas & 20 & 33,9 \\
\hline \multicolumn{3}{|c|}{ Resolução do problema de saúde bucal $(n=59)^{* * *}$} \\
\hline Totalmente & 27 & 45,8 \\
\hline Parcialmente & 21 & 35,6 \\
\hline Não & 11 & 18,6 \\
\hline \multicolumn{3}{|c|}{ Dificuldade de agendar consulta odontológica na gestação $(n=59)^{\star * *}$} \\
\hline Sim & 4 & 6,8 \\
\hline Não & 55 & 93,2 \\
\hline \multicolumn{3}{|c|}{ Oportunidade de expressar suas queixas na consulta odontológica $(n=58)^{* * *}$} \\
\hline Sim & 47 & 81,0 \\
\hline Não & 11 & 19,0 \\
\hline \multicolumn{3}{|c|}{ Oportunidade de opinar sobre o tratamento odontológico $(n=57)^{* * *}$} \\
\hline Sim & 26 & 45,6 \\
\hline Não & 31 & 54,4 \\
\hline \multicolumn{3}{|c|}{$\begin{array}{l}\text { 'Apesar da amostra total, para algumas questões houve perdas (indivíduo se recusou a responder a pergunta ou relatou } \\
\text { não saber respondê-la) durante o processo de coleta de dados, o que resultou em "n" menores do que o que era possível, } \\
\text { para algumas "variáveis". } \\
\text { *Todas as mulheres investigadas eram aptas a fornecer essa informação (268). } \\
{ }^{* *} \text { O total de mulheres aptas a responder essa informação, representa aquelas que afirmaram ter algum contato com o } \\
\text { dentista durante a gestação (68). } \\
\text { ***O total de mulheres aptas a responder essa informação, representa aquelas que afirmaram ter realizado consulta com } \\
\text { o dentista durante a gestação (59). }\end{array}$} \\
\hline
\end{tabular}


Tabela 3: Caracterização do pré-natal odontológico na Atenção Básica: participação em ações educativas individuais e coletivas. Vitória da Conquista-BA. Brasil. 2013-20141.

\begin{tabular}{llc}
\hline VARIAVÉIS & $\mathbf{n}$ & $\%$ \\
\hline Participação em ação educativa com o dentista durante a gestação & & \\
$(\mathbf{n}=67)^{*}$ & 12 & 17,9 \\
Sim & 55 & 82,1
\end{tabular}

Participação em ação educativa com o dentista exclusivamente para gestantes $(n=12)^{* \star}$

Sim

Não

$5 \quad 41,7$

Avaliação da ação educativa ministrada pelo dentista $(n=11)^{* *}$ Satisfatória

Possibilidade de emitir opinião na ação educativa $(n=12)^{\star *}$

Sim

Não

Saiu da ação educativa sem conseguir tirar dúvidas $(n=12)^{\star *}$

Foi orientado pelo dentista sobre o aleitamento materno $(n=66)^{*}$

Sim

Foi orientado pelo dentista sobre chupeta ortodôntica $(n=66)^{*}$

Não

Foi orientado pelo dentista sobre quando fazer higiene bucal da criança $(n=66)^{*}$

Não

Foi orientado pelo dentista sobre como fazer higiene bucal da criança $(n=66)^{*}$

Sim

Não

Foi orientado pelo dentista sobre higiene bucal da criança após o uso de xaropes $(n=66)^{*}$

Foi orientado pelo dentista sobre a oferta de açúcar/mel para a criança $(n=66)^{*}$
Sim

1 Apesar da amostra total, para algumas questões houve perdas (indivíduo se recusou a responder a pergunta ou relatou não saber respondê-la) durante o processo de coleta de dados, o que resultou em "n" menores do que o que era possível, para algumas variáveis". * $O$ total de mulheres aptas a responder essa informação, representa aquelas que afirmaram ter algum contato com o dentista durante a gestação (68).

** Essas variáveis referem-se às ações educativas coletivas. O total de mulheres aptas a responder essas informações, representa aquelas que afirmaram ter participado de ação educativa com o dentista durante a gestação (12). 
Outros autores também verificaram a baixa cobertura de pré-natal odontológico ${ }^{14,15,16,17,18,19}$. Por outro lado, estudos desenvolvidos na Colômbia ${ }^{20,21}$ demonstram que os programas de incentivo à saúde bucal da gestante podem influenciar nas amplas coberturas de prénatal odontológico observadas, que chegou a $85 \%$, a despeito das iniquidades identificadas no acesso ao mesmo ${ }^{21}$. No Brasil, apesar da atenção à saúde bucal da gestante ter sido mencionada em importantes políticas de atenção a esse grupo ${ }^{11,12}$, no cotidiano dos serviços de saúde ainda é dispensada pouca atenção à essa linha de cuidado ${ }^{10}$, o que ficou claro nos resultados dessa pesquisa. Outros dados apontam que em países em desenvolvimento apenas 30 a $55 \%$ das gestantes receberam atenção odontológica, enquanto em países desenvolvidos 0 quantitativo dessas mulheres chega entre 70 a $90 \%{ }^{22}$.

Os resultados aqui apresentados são baseados em mulheres regularmente inscritas no programa de pré-natal das unidades de saúde da Atenção Básica. Tais unidades foram selecionadas para a pesquisa por contarem com a presença de equipe de saúde bucal. Apesar disso, a baixa cobertura de pré-natal odontológico foi uma realidade, o que cria a necessidade de elucidar os fatores que a condicionam, num cenário de presença de serviço odontológico. Nesse sentido, outro estudo pode ajudar nessa compreensão, ao apontar três conjuntos de fatores que têm gerado barreiras ao pré-natal odontológico: fatores relacionados ao dentista, fatores relativos aos profissionais envolvidos no prénatal e fatores referidos pela gestante ${ }^{23}$.

No modelo atual de Atenção Básica, a interdisciplinaridade e o trabalho interprofissional surgem como elementos-chave norteadores do processo de trabalho, e importantes ferramentas para consolidar o pré-natal odontológico. Portanto, a falta de integração da ESB com os demais profissionais que compõem a equipe responsável pelo acompanhamento da gestante no pré-natal, compreende, nos dias de hoje, uma falha a ser superada ${ }^{15}$. Assim, por um lado, como apontam Codato e Nakama ${ }^{3}$, é fundamental que dentistas não se limitem apenas ao trabalho técnico-odontológico, e extrapolem os limites da cavidade bucal, com interação e trocas de saberes com profissionais de outras áreas, visando à integralidade da atenção à gestante. Assim, a integração do dentista, como membro da equipe de pré-natal, através de dispositivos, comoasagendascompartilhadas, interconsultas e projetos terapêuticos singulares, resulta em mais conforto e melhor atenção à gestante, em todos os níveis: educativos, preventivos e curativos.

Por outro lado, a literatura aponta que, muitas vezes, médicos e enfermeiros dão pouca importância aos cuidados necessários à saúde oral da gestante ${ }^{10}$. Santos-Neto et al. ${ }^{19}$ num estudo com 1.035 puérperas, verificou que apenas $11,7 \%$ afirmaram que receberam assistência odontológica adequada, 21,2\% foram orientadas pelo enfermeiro sobre assistência odontológica na gestação e somente $18,2 \%$ foram orientadas pelos médicos.

Contudo, muitos dentistas, médicos, enfermeiros e gestantes ainda sustentam dúvidas, cientificamente infundadas, quanto à atenção odontológica durante a gestação e, diante disso, fortalecem mitos e tabus, que por um lado promovem o afastamento de gestantes do serviço odontológico, e por outro, levam os profissionais a protelarem os cuidados em saúde bucal a esse grupo para o período pós-parto ${ }^{10,24}$. Ao incluir o dentista nesse escopo, a realidade se torna ainda mais complexa, pois este deveria ser o principal agente de mudança e desconstrução de tais mitos. Acredita-se que problemas na formação profissional do dentista, com pouca abordagem 
do cuidado à gestante, podem favorecer a manutenção da insegurança em lidar com este público. Somando-se a isso, acredita-se que o profissional, em algumas situações, pode se esquivar deste tipo de ação por medo de ser responsabilizado, caso haja intercorrência com a gestante, mesmo que esta não tenha resultado da intervenção odontológica. Por fim, acredita-se que a falta de inserção da saúde bucal enquanto prioridade seja merecedora de destaque, o que a situa muito mais no campo do tratamento e não na dimensão de cuidado integral, algo que fica evidente quando os dados apontam a grande procura do dentista por motivo de dor.

A baixa cobertura do pré-natal odontológico também pode resultar da atitude de afastamento ou negligência pela própria mulher. Os fatores referidos pelas gestantes foram, segundo Moimaz et al. ${ }^{16}$ : não havia necessidade de tratamento $(32,9 \%)$; mitos, crendices e tabus $(16,4 \%)$; falta de dinheiro, vontade e /ou tempo $(15,1 \%)$; medo $(8,2 \%)$; ida ao dentista antes da gravidez (11\%); problemas nos postos de saúde $(9,6 \%)$; orientação do médico a não tratar $(1,4 \%)$; não teve motivo $(2,7 \%)$ e outros $(2,7 \%)$.

Para alguns autores, as principais variáveis que guardaram relação com maior cobertura de pré-natal odontológico, após ajustes por potenciais confundidores, foram: mulheres casadas, maior nível de escolaridade, acompanhamento pré-natal na rede privada, primiparidade e maior renda ${ }^{14,21}$. A amostra avaliada nesta pesquisa, numa perspectiva descritiva demonstrou características diferentes das citadas pelo autor anteriormente referido, exceto para situação conjugal. Apesar disso, a metodologia utilizada nessa investigação não possibilita fazer tais associações.

Cabe destacar que a amostra desse estudo demonstrou ser composta por mulheres que relataram baixa procura por serviço odontológico, mesmo antes do período gestacional. Adicionalmente, a condição especial da gestação pouco contribuiu para busca por atenção em saúde bucal, apesar das necessidades peculiares a esse grupo específico, uma vez que o percentual de mulheres que não fizeram uso regular do serviço odontológico, das unidades de saúde, foi levemente maior do que a quantidade de mulheres que não usaram esse serviço, no período gestacional. Em algumas situações, a elevação da procura se dá não pela conscientização em relação à importância do pré-natal odontológico, mas pela prioridade de atendimento durante a gestação, que traz mais facilidade na resolução de problemas que eram crônicos, e para os quais não houve possibilidade de assistência antes da gestação ${ }^{3}$.

Além disso, nas situações em que houve contato da gestante com a ESB, a dor foi o principal motivo que impulsionou essa demanda, enquanto uma menor parte relatou a procura para iniciar tratamentos em casos sem sintomatologia dolorosa, ou buscar ações preventivas. Isso reflete o caráter emergencial dado ao contato dentista-gestante, também evidenciado por Moimaz et al. ${ }^{16,18}$, o que pode fortalecer a alienação das gestantes em relação à sua saúde bucal, naturalizando, como algo inerente da gestação ou da vida, processos patológicos e fatores de risco que deveriam ser abordados através do pré-natal odontológico.

Apesar de representarem uma minoria (68; $25,4 \%$ ), no tocante ao total de mulheres estudadas, dentre aquelas que receberam atenção odontológica, uma parcela considerável relatou resolubilidade dos problemas bucais, mostrando que a Atenção Básica pode ser um espaço efetivo para consolidação do cuidado em saúde bucal. Entretanto, foi também significativo o total de mulheres que relatou a resolubilidade parcial dos problemas bucais, que pode estar relacionada à demanda por procedimentos 
de maior complexidade, com dificuldade de retaguarda do serviço especializado, ou a espera para realização da intervenção num momento oportuno de maior comodidade para a própria gestante. Entretanto, conforme assinalam Martins et al. ${ }^{24}$ e Codato et al.$^{25}$, esse fato pode resultar da iniciativa de postergar tratamentos mais complexos para o período pós-gestacional, após alívio dos sintomas agudos, por insegurança dos profissionais.

Cabe também destacar, nos resultados aqui apresentados, que dentre as mulheres que tiveram acesso à atenção odontológica na gestação, a maior parcela relatou oportunidade de expressão das suas queixas, o que denota algo positivo na abordagem profissional, que favorece a incorporação da subjetividade no planejamento da ação clínica, sobretudo numa fase de maior sensibilidade da mulher. Entretanto, em outro relato da literatura ${ }^{26}$, assim como em nossos achados, para a maioria das gestantes, a decisão final do tratamento ainda ocorre de maneira unilateral, excluindo o paciente do processo, que no caso da gestante, pode aumentar os níveis de insegurança.

Nos resultados apresentados, é notória a ausência de participação das mulheres em ações educativas, dentre aquelas que relataram algum contato com o serviço odontológico (12; $17,9 \%)$. Tais atividades também compõem as atribuições sob responsabilidade da ESB no pré-natal. Ações dessa natureza são importantes para ajudar a gestante a identificar, compreender e lidar com as alterações bucais que podem ser visualizadas durante a gestação. Entretanto, as estratégias educativas são ferramentas indispensáveis na sensibilização de gestantes quanto à importância do tratamento odontológico, inclusive contribuindo para acolhimento, ampliação do vínculo, redução da insegurança da gestante e desmistificação de tabus ainda sustentados pelas mesmas.
Comautilizaçãodeestratégiaseducacionais é possível trabalhar questões importantes para o cuidado com a saúde bucal do bebê, através da abordagem de temas como aleitamento materno, utilização racional do açúcar, cuidados com medicamentos açucarados, uso de bicos artificiais, importância da dentição decídua e limpeza da cavidade oral ${ }^{26}$. No grupo pesquisado, verificou-se que houve predomínio de mulheres que não receberam orientações sobre tais aspectos, os quais para serem abordados não dependem exclusivamente da realização de grupos operativos, pois podem também ser trabalhados na consulta individual da gestante. No entanto, a maior parte das ações educativas observadas parece ter se resumido à orientação da higiene oral do bebê.

Há relato na literatura de maior acesso às orientações sobre saúde bucal na gravidez, no qual observou $\square$ se que $41,66 \%$ das gestantes receberam algum tipo de orientação e, dentre estas, $80 \%$ receberem informações através da equipe de saúde bucal ${ }^{15}$, que apesar de não chegar à maioria das mulheres, demonstrou números mais significativos do que aquele observado nessa investigação. A futura mãe tem papel fundamental na disseminação de hábitos saudáveis no seio da família e representa um importante agente na promoção de saúde, além disso, a gestação, por si só, eleva o sentimento de preservação da espécie, e sensibiliza a mãe à apreensão de novos conhecimentos e atitudes, que possibilitem maior qualidade de vida ao bebê ${ }^{16}$. Esse argumento justifica a importância e dá um caráter imperativo à atenção odontológica para as gestantes, através da educação em saúde.

É também importante mencionar, mesmo com a identificação de mínima participação das gestantes em ações educativas, que a participação nas ações de educação em saúde com dentistas, em muitas situações, não possibilitou a emissão de opinião sobre os temas abordados, embora as gestantes 
relataram que conseguiram respostas para as dúvidas. A possibilidade de ser ouvida pelos profissionais, ainda que apenas para responder às dúvidas existentes, pode ter contribuído para que grande parte das mulheres tenha julgado a atividade educativa como satisfatória. É possível, embora nossa metodologia não nos permita fazer tal afirmação, que ao possibilitar que as gestantes verbalizassem dúvidas, o profissional tenha elevado os níveis de confiança e segurança das mesmas.

Nesse sentido, cabe uma reflexão sobre o modelo de educação em saúde que ainda permeia, nos dias atuais, as práticas em saúde, em alguns espaços, às quais se dão com a desvalorização do saber popular, que permanece subjugado ao saber científico e, sobretudo, clínico. Além disso, ressalta-se que as metodologias utilizadas neste cenário se baseiam na mera transmissão de informações, sem de fato haver preocupação de como elas são apreendidas pelo público-alvo, ou até que ponto tais informações cabem no contexto de vida do mesmo ${ }^{27}$. Sem o diálogo necessário para fazer a interlocução entre as recomendações dos profissionais e os aspectos culturais, muitas vezes transmitidos entre gerações, há grandes possibilidades de haver uma tensão na tomada de decisões que interferem na saúde, que pode dificultar a adoção de comportamentos saudáveis.

Por outro lado, é importante utilizar o conhecimento fragmentado e pouco elaborado do educando como matéria-prima e, através da valorização do saber e valores do mesmo, permitir que ele se sinta "em casa" e mantenha sua iniciativa na busca de conhecimento. A partir disso, não apenas o conteúdo discutido é revolucionário, mas há horizontalidade na discussão, permeada pela ampliação dos espaços de interação cultural e negociação entre os diversos atores, para a construção compartilhada do conhecimento ${ }^{28}$. Assim, acredita-se que por se tratar de um público-alvo tão cercado de mitos e arraigado em aspectos culturais, as gestantes devam participar de processos educativos inovadores e dialógicos.

Por fim, cabe salientar que para a interpretação dos resultados aqui apresentados deve-se considerar que os mesmos foram obtidos num grupo de mulheres com gestação em andamento, estratégia utilizada para redução de viés de memória. Embora a maioria já estivesse em fases mais avançadas da gestação, e a recomendação seja de inserção no pré-natal odontológico logo após a identificação da gravidez, estes resultados não permitem definir o tipo de atenção odontológica recebido até o fim do período gestacional. Além disso, o resultado é válido para as unidades de saúde pesquisadas. De forma semelhante, se refere à população de mulheres que buscaram, durante o mês em que a coleta foi desenvolvida em suas unidades de referência, consulta pré-natal ou puerperal. Quanto a isso, acredita-se que a maioria das mulheres, regularmente acompanhadas no pré-natal/puerpério, procuraria a unidade de saúde dentro desse período. Novas pesquisas devem ser realizadas com profissionais envolvidos no pré-natal, além da utilização de metodologias analíticas e qualitativas, para melhor compreender a configuração da realidade aqui apresentada.

\section{Conclusão}

Diante dos resultados aqui apresentados e discutidos, é plausível concluir que o pré-natal odontológico não se apresentou como prática consolidada, pois a maior parte das mulheres estudadas não estabeleceu contato com dentista durante a gestação. Além disso, nos casos em que este contato foi estabelecido, não resultou de uma linha de cuidado organizada para este ciclo vital, pois muitas vezes esteve condicionado à resolução de sintomatologia dolorosa, e não estabeleceu uma dimensão 
de cuidado mais integral e longitudinal. Houve baixa participação das mulheres investigadas em ações clínicas assistenciais, de prevenção ou de promoção da saúde bucal, durante a gestação, mesmo com poucos relatos de dificuldades para agendamento de consultas. Apesar disso, nos casos de consulta com o dentista, essas frequentemente se deram numa frequência de até duas vezes na gestação, com algum grau de resolubilidade da queixa apresentada pela mulher.

A maioria das gestantes não foi orientada sobre os principais tópicos de saúde bucal, referentes aos cuidados com o bebê, tais como: vantagens do aleitamento materno, uso e implicações da chupeta ortodôntica, quando e como realizar a higiene bucal do bebê, cuidados no uso de xaropes açucarados e oferta de açúcar/mel. Por fim, cabe destacar que, ao participar de ações educativas desenvolvidas, as mulheres relataram não ter oportunidade para emitir suas opiniões, sobre os temas debatidos.

É necessário ampliar as discussões em torno do pré-natal odontológico e da integração da equipe de saúde bucal aos demais profissionais responsáveis pelo acompanhamento pré-natal. Nesse sentido, a formação em Odontologia precisa subsidiar e estimular a realização dessa prática, na dimensão promotora de saúde, preventiva e assistencial. Além disso, as tecnologias relacionais precisam ser colocadas no cerne da linha de cuidado da gestante, dada às questões culturais peculiares à mesma, que, muitas vezes, pode ser colocar como importante barreira à adesão ao pré-natal odontológico. Por fim, salienta-se a importância da educação permanente nos serviços de saúde, para subsidiar o processo de trabalho da equipe de saúde, e fomentar a integração da mesma, fortalecendo o cuidado pré-natal com base na integralidade.

\section{REFERÊNCIAS}

1 Brasil. Ministério da Saúde. Secretaria de Atenção à Saúde. Departamento de Atenção Básica. Saúde Bucal. Brasília, DF; 2008.

2 Gaffield ML, Gilbert BJ, Malvitz DM, Romaguera $R$. Oral health during pregnancy: na analysis of information collected by the pregnancy risk assessment monitoring system. J Am Dent Assoc. 2001; 132: 1009-16.

3 Codato LAB, Nakama L, Melchior R. Percepções de gestantes sobre atenção odontológica durante a gravidez. Ciênc. saúde coletiva. 2008; 13(3): 1075-80.

4 Moreira PVL, Chaves AMB, Nóbrega MSC. Uma Atuação Multidisciplinar Relacionada à Promoção de Saúde. Pesq Bras Odontoped Clin Integr. 2004; 4(3): 259-64.

5 Elias R, Figueredo MA, Yurguel L. Atendimento estomatológico em gestantes - verdades e mitos. Rev ABO Nac. 2003/04; 11(6 Suppl): 333-4.

6 Vieira, DRP, Feitosa DMZ, Alves MSC, Cruz MCFN, Lopes FF. Associação entre doença periodontal na gravidez e parto pré-termo baixo peso ao nascer. Odontol Clín Cient. 2010; 9(4): 311-14.

7 Moimaz SAS, Saliba O, Santos KT, Queiroz APDG, Garbim CAS. Prevalência de Cárie Dentária em Gestantes Atendidas no Sistema Único de Saúde em Município Paulista. Rev Odontol Araçatuba. 2011; 32(1): 44-8.

8 Vries MRA, Aires DFLM, Groisman S, Toledo E. Perfil das Gestantes do pré-natal do Hospital Maternidade de Nova Friburgo (HMNF). Rev Perio News. 2008; 2(2): 147-52.

9 Reis DM, Pitta DR, Ferreira HMB, Jesus MCP de, Moraes MEL de. Educação em saúde como estratégia de promoção de saúde bucal em gestantes. Ciênc saúde coletiva. 2010; 15(1): 269-76.

10 Leal NP, Janotti CB. Saúde bucal da gestante atendida pelo SUS: práticas e representações de profissionais e pacientes. Femina. 2009; 37(8): 413-21

11 Brasil. Ministério da Saúde. Secretaria de Atenção à Saúde. Departamento de Atenção Básica. Diretrizes da Política Nacional de Saúde Bucal. Brasília, DF; 2004. 
12 Brasil. Ministério da Saúde. Secretaria de Atenção à Saúde. Departamento de Atenção Básica. Atenção ao pré-natal de baixo risco: Caderno da Atenção Básica $n^{\circ} 32$. Brasília, DF; 2012.

13 Mameluque S, Souza-Júnior EB, Rezende JC, Costa CCG, Vanham IM, Oliveira JM, et al. Abordagem integral no atendimento odontológico à gestante. Unimontes Cient. 2005; 7(1): 67-75.

14 Kloetzel MK, Huebner CE, Milgrom P. Referrals for Dental Care During Pregnancy. J midwifery womens health. 2011; 56(2): 110-17.

15 Alves CS, Bezerra MM. Atenção odontológica no pré-natal: a percepção das gestantes do bairro Padre Palhano, Sobral-CE. Sanare 2005; 7(1): 61-8.

16 Moimaz SAS, Rocha NB, Saliba O, Garbin CAS. O acesso de gestantes ao tratamento odontológico. Rev odontol univ cid São Paulo. 2007; 19(1): 39-45.

17 Praetzel JR, Ferreira FV, Lenzi TL, Melo GP, Alves LS. Percepção materna sobre atenção odontológica e fonoaudiológica na gravidez. Rev gaúcha odontol. 2010; 58(2): 155-60.

18 Moimaz SAS, Garbin CAS, Rocha NB, Santos SMG, Saliba NA. Resultados de dez anos do programa de atenção odontológica à gestante. Rev ciênc ext. 2010; 7(1): 42.

19 Santos-Neto ET, Oliveira AE, Zandonade E, Leal MC. Acesso à assistência odontológica no acompanhamento pré-natal. Ciênc saúde colet. 2012; 17(11): 3057-68.

20 Saldarriaga OJ, Sánchez M, Avendaño L. Conocimientos y prácticas em salud bucal de las gestantes vinculadas al programa de control prenatal. Rev CES odontología. Medellín 2003; 17(2).

21 Corchuelo-ojeda J, Pérez GJG. Determinantes socioeconómicos de laatención odontológica durante lagestaciónen Cali, Colombia. Cad saúde pública. 2014; 30(10): 2209-18.

22 Lopez NJ, Smith PC, Gutierrez J. Higher risk of preterm birth and low birth weight in women with periodontal disease. J dent res. 2002; 81: 58-63.

23 Soares MRPS, Dias AM, Machado WC, Chaves MGAM, Chaves-Filho HDM. Pré-natal odontológico: a inclusão do cirurgião-dentista nas equipes de pré-natal. Rev inter de estudos experimentais. 2009; 1(2): 53-7.

24 Martins LO. Assistência odontológica à gestante: percepção do cirurgião-dentista. Rev pan-amaz saude. 2013; 4(4): 11-8.

25 Codato LAB, Nakama L, Cordoni-Júnior L, Higasi MS. Atenção odontológica à gestante: papel dos profissionais de saúde. Ciênc saúde coletiva. 2011; 16(4): 2297-301.

26 Reis DM, Pitta DR, Ferreira HMB, Jesus MCP de, Moraes MEL de. Educação em saúde como estratégia de promoção de saúde bucal em gestantes. Ciênc saúde coletiva. 2010; 15(1): 269-76.

27 Mialhe FL, Silva CMC. A educação em saúde e suas representações entre alunos de um curso de odontologia. Ciênc saúde coletiva. 2011; 16(Suppl. 1): 1555-61.

28 Vasconcelos EM. Educação popular: de uma prática alternativa à uma estratégia de gestão participativa das políticas de saúde. Physis (Rio J.). 2004; 14(1): 67-83.

Submetido em: 20-12-2015

Aceito em: 24-8-2016 